\title{
Relationship between aspect ratio and suspension viscosity of wood cellulose nanofibers
}

\author{
Shinichiro Iwamoto, Seung-Hwan Lee ${ }^{1}$ and Takashi Endo \\ Cellulose nanofibers (CNFs) with aspect ratios varying from 30-300 were prepared from wood by acid hydrolysis and \\ mechanical disintegration. The length and width of the individual CNFs were measured from atomic force microscopy (AFM) \\ images. The steady shear properties of the CNF suspensions were measured using cone-plate type and capillary rheometers to \\ determine their intrinsic viscosities. Higher aspect ratio CNFs had larger intrinsic viscosity values. The predicted aspect ratios \\ on the basis of rigid rod theory for the lowest aspect ratio CNFs corresponded well with our measured values. However, for \\ higher aspect ratio CNFs, the predicted values did not agree with the experimental values. A comparison of the CNF intrinsic \\ viscosities with other types of particles and polymers indicated that the CNFs are semi-flexible rods rather than rigid rods. \\ Polymer Journal (2014) 46, 73-76; doi:10.1038/pj.2013.64; published online 17 July 2013
}

Keywords: aspect ratio; cellulose nanofibers; viscosity; wood

\section{INTRODUCTION}

Cellulose, the main constituent of wood, is found in $3-4 \mathrm{~nm}$ thick fibers in plant cell walls. Native nano-scale cellulose can be obtained from wood by chemical and mechanical treatments. Rod-like cellulose nano-crystals (CNCs) with thicknesses and lengths of 3-10 nm and $100-300 \mathrm{~nm}$, respectively, have been obtained by acid hydrolysis. The low aspect ratio of CNCs $(<100)$ has facilitated detailed analysis of their shapes. ${ }^{1-4}$ Mechanical disintegration can be used as an alternative to acid hydrolysis to avoid cellulose degradation, allowing the isolation of large aspect ratio native cellulose nanofibers (CNFs). The resulting CNFs have been reported to be as thin as 3-15 nm. ${ }^{5-9}$ However, measuring their lengths has been difficult because of their elongated shapes. ${ }^{10,11}$

Nanomaterials shapes are inherently difficult to determine because of their small dimensions. Shape determination becomes even more difficult for nanomaterials with higher isotropic dimensions, such as nanofibers, nanotubes and nanowires. Visualization of nanomaterials typically requires the use of electron microscopy or atomic force microscopy (AFM). Observation of anisotropic nanomaterials usually does not provide shape information. Owing to the use of small observational fields, the time and effort required to perform detailed shape observations would be prohibitive. Therefore, it is desirable to use shape-correlating properties (for example, viscosity and lightscattering profile) to characterize the shapes of nanomaterials.

The aspect ratio (that is, length/diameter) of a nanomaterial influences its viscosity in suspension. For CNC water suspensions, the viscosity can be described as a function of the $\mathrm{CNC}$ aspect ratio using rigid rod theory. ${ }^{2,12}$ However, this theory has not been experimentally tested for CNFs and the conformational properties of these materials have not been well studied.

In the present study, we analyzed the conformation of CNFs using their intrinsic viscosities in suspension. CNCs and CNFs with aspect ratios of 30-300 were prepared by acid hydrolysis and mechanical disintegration. The lengths and widths of the CNCs and CNFs were measured by AFM. The samples used for AFM were prepared from highly dilute suspensions with careful examination to accurately define the lengths. The intrinsic viscosities of the $\mathrm{CNC}$ and $\mathrm{CNF}$ water suspensions were measured to describe the relationship between intrinsic viscosity and aspect ratio for CNCs and CNFs.

\section{MATERIALS AND METHODS}

Preparation of CNFs

CNCs were prepared from wood cellulose powder (KC Flock, W-400G, Nippon Paper Chemicals Co. Ltd, Tokyo, Japan). The wood cellulose powder was hydrolyzed for $4 \mathrm{~h}$ at $60^{\circ} \mathrm{C}$ with $50 \mathrm{wt} \%$ sulfuric acid. The hydrolyzed powder was washed and centrifuged in distilled water four times and dialyzed for 4 days. The hydrolyzed cellulose suspension was then separated into sediment and supernatant fractions by centrifugation at $1200 \mathrm{~g}$ for $5 \mathrm{~min}$. The supernatant fractions were sonicated in an ice bath for $15 \mathrm{~min}$ using an ultrasonic homogenizer equipped with a 3-mm $\phi$ tip (US-150T, Nissei Corp., Tokyo, Japan). Sonication was repeated another five times for $3 \mathrm{~min}$ with 2-min intermissions between each repetition. Finally, the unpulverized cellulose was removed by centrifugation at $30000 \mathrm{~g}$ for $10 \mathrm{~min}$ to obtain the CNC suspension.

Biomass Refinery Research Center (BRRC), National Institute of Advanced Industrial Science and Technology (AIST), Higashihiroshima, Japan

${ }^{1}$ Current address: Department of Forest Biomaterials Engineering, College of Forest and Environmental Sciences, Kangwon National University, 1 Kangwondaehak-gil, Chuncheon-si, Gangwon-do, 200-701, Republic of Korea

Correspondence: Dr S Iwamoto, Biomass Refinery Research Center, National Institute of Advanced Industrial Science and Technology, 3-11-32, Kagamiyama, Higashihiroshima, Hiroshima 739-0046, Japan.

E-mail: s.iwamoto@aist.go.jp

Received 2 April 2013; revised 21 May 2013; accepted 27 May 2013; published online 17 July 2013 
CNFs were prepared from milled Japanese cypress softwood. Holocellulose was obtained by delignification of wood powder according to a previous report. ${ }^{7}$ Briefly, delignification of the aqueous powder suspension was achieved by five cycles of sodium chlorite $\left(\mathrm{NaClO}_{2}\right)$ treatment for an hour under acidic conditions ( $\mathrm{pH} 4-5)$ at $70^{\circ} \mathrm{C}$. The holocellulose was fibrillated using a highpressure homogenizer (MMX-L200-D10, Masuko Corp., Saitama, Japan) set at $200 \mathrm{MPa}$ to obtain the CNFs. The aqueous holocellulose suspension (3wt\%) was passed through the homogenizer 10 times.

The CNF water suspension $(0.5 \mathrm{wt} \%, 50 \mathrm{ml})$ was sonicated in an ice bath for an hour with an ultrasonic homogenizer equipped with a $20-\mathrm{mm} \phi$ tip to shorten the nanofiber lengths, according to the report of Li and Renneckar. ${ }^{11}$ Sonication was repeated 20 times for $3 \mathrm{~min}$ with an intermission of $2 \mathrm{~min}$ between each repetition. The shortened CNFs are hereafter referred to as SCNFs,

\section{Atomic force microscopy}

The dilute CNF suspension (0.001-0.0001 wt\%) was deposited on mica precoated with polyethyleneimine. The suspension was adsorbed on the mica surface for $5 \mathrm{~min}$ under ambient conditions, rinsed with distilled water and dried by air blowing. The polyethyleneimine acted as an absorbent of the CNFs onto the mica surface. The nanofibers were examined by contact mode AFM (JSPM-5200S, JEOL Ltd., Tokyo, Japan) using triangle-shaped DNP-10 (Veeco Inc., Plainview, NY, USA) cantilevers (spring constant, $0.06 \mathrm{~N} \mathrm{~m}^{-1}$ ). Nanofiber length and thickness were measured from AFM images using image-analyzing software included with the AFM system. The nanofiber thickness was defined as the height difference between the mica surface and the top surface of the nanofiber.

\section{Zeta potentials}

CNF zeta potentials were measured in water by the laser Doppler method using a nanoparticle analyzer (SZ-100, Horiba Ltd., Kyoto, Japan). The concentration of the samples was $0.001 \mathrm{wt} \%$. The measurements were performed at $25^{\circ} \mathrm{C}$.

\section{Viscosity of the water suspensions}

The steady shear properties of the CNF and SCNF suspensions were measured using a cone-plate type rheometer (AR-G2, TA Instruments, New Castle, DE, USA) at a shear rate of $0.1-100 \mathrm{~s}^{-1}$ at $20^{\circ} \mathrm{C}$. The cone diameter and angle were $60 \mathrm{~mm}$ and $1^{\circ}$, respectively. Water evaporation was prevented using a specially designed jacket on the rheometer. The measurements were performed from low to high shear rates. The CNC suspension viscosities were measured using a capillary viscometer (0c type, Ubbelohde viscometer, Lauda, Lauda-Königshofen, Germany). The water flow time was $357 \mathrm{~s}$.

\section{RESULTS AND DISCUSSION}

\section{Morphologies and surface charges of the CNFs}

The CNCs, SCNFs and CNFs were observed by AFM to determine their thicknesses and lengths. Representative AFM images are shown in Figure 1. The sulfuric acid treatment preferentially hydrolyzed the amorphous regions of the cellulose. The CNCs collected from the acid hydrolysis residues were rod-like cellulose crystals absent of kinks and curves. The lengths of the SCNFs were shorter than the lengths of the CNFs, indicating that sonication treatment decreased the fiber length.

The thickness and length values for more than 50 fibers are plotted in Figure 2. The average values and aspect ratios are listed in Table 1. The CNCs were thicker than the SCNFs and CNFs, indicating that the CNCs were composed of bundles of cellulose crystals. The wide distribution of $\mathrm{CNC}$ thickness values was due to incomplete peeling of the crystals from the bundles. The thicknesses of the SCNFs and CNFs were almost the same and corresponded to the thickness of wood cellulose crystals. The CNCs were shorter than the other samples. The measured aspect ratios increased in the order $\mathrm{CNFs}>\mathrm{SCNFs}>\mathrm{CNCs}$.

The CNCs, SCNFs and CNFs dispersed well in water. It was difficult to sediment the nanofibers from their water suspension, even after centrifugation. The CNCs dispersed well in water because of the sulfate ester groups on their surfaces. The sulfuric acid hydrolysis of the cellulose substituted some of hydroxyl groups with sulfate ester groups. The SCNFs and CNFs dispersed well in water because of surface carboxylic acid groups that were introduced during delignification. ${ }^{13}$

The sulfate ester and carboxylic acid groups imparted negative charges to the nanofibers in water. To assess the charge level, the zeta potentials of the CNC, SCNF and CNF water suspensions were measured. The zeta potentials for the CNCs, SCNFs and CNFs were $-65,-64$ and $-63 \mathrm{mV}$, respectively. Although the negative charges were derived from different functional groups, the charge levels of the CNCs, SCNFs and CNFs were almost same.

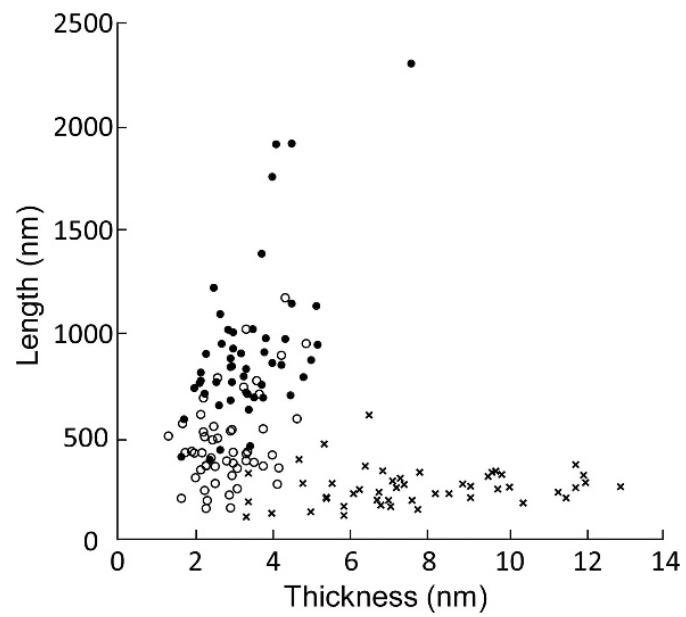

Figure 2 Size distributions of cellulose nano-crystals (CNCs; cross, $\times$ ), shortened cellulose nanofibers (SCNFs; open circle, $\bigcirc$ ) and cellulose nanofibers (CNFs; filled circle,
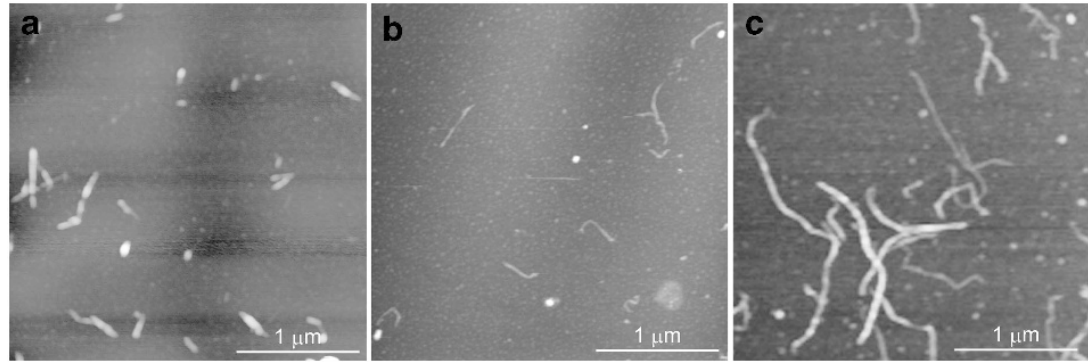

Figure 1 Atomic force microscopy (AFM) images of (a) cellulose nano-crystals (CNCs), (b) shortened cellulose nanofibers (SCNFs) and (c) cellulose nanofibers (CNFs). 
Table 1 Shapes of CNCs, CNFs, and SCNFs measured by AFM, their intrinsic viscosities, and aspect ratios predicted by the Simha equation ${ }^{14}$

\begin{tabular}{lcrccc}
\hline & $\begin{array}{c}\text { Thickness } \\
(\mathrm{nm})^{\mathrm{a}}\end{array}$ & \multicolumn{1}{c}{$\begin{array}{c}\text { Length } \\
(\mathrm{nm})^{\mathrm{a}}\end{array}$} & $\begin{array}{c}\text { Aspect } \\
\text { ratio }^{\mathrm{a}}\end{array}$ & $\begin{array}{c}\text { Intrinsic viscosity } \\
{[\eta]}\end{array}{\left.\mathrm{m} / \mathrm{g}^{-1}\right)}^{\text {Predicted }}$ & $\begin{array}{c}\text { Pspect ratio } \\
\text { CNCs }\end{array}$ \\
\hline SCNFs & $3.4 \pm 2.5$ & $291 \pm 102$ & $47 \pm 24$ & 91 & 45 \\
CNFs & $3.7 \pm 1.2$ & $573 \pm 249$ & $207 \pm 81$ & 677 & 140 \\
\hline
\end{tabular}

Abbreviations: AFM, atomic force microscopy; CNC, cellulose nano-crystal; CNF, cellulose nanofiber; SCNF, shortened cellulose nanofiber.

Weighted mean values measured using AFM.
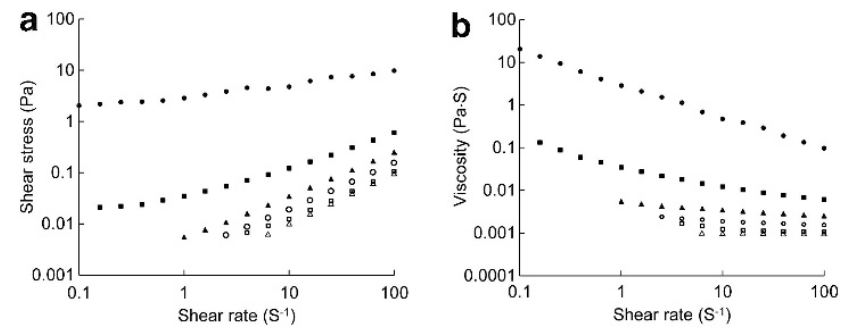

Figure 3 Shear stress (a) and viscosity (b) values of cellulose nanofiber (CNF) suspensions of various concentrations and water; filled circles ( $)$ $0.5 \mathrm{wt} \%$, filled squares (口) $0.3 \mathrm{wt} \%$, filled triangles (A) $0.1 \mathrm{wt} \%$, open circles ( $\bigcirc)$ ) 0.05 wt\%, open squares $(\square) 0.01$ wt\% and open triangles $(\Delta)$ water.

\section{Viscosities of the CNF suspensions}

Figure 3 shows the shear stress and viscosity values of the CNF suspensions and water under steady state flow. Shear thinning was clearly observed at a concentration of more than $0.3 \mathrm{wt} \%$ over the entire shear rate range. Viscosities decreased with increases in shear rate. The observed shear thinning was caused by interactions between CNFs orientated along the direction of shear. In the high shear rate region $\left(>10 \mathrm{~s}^{-1}\right)$ of the low-concentration suspension $(<0.1 \mathrm{wt} \%)$, shear thinning gradually decreased and approximated Newtonian behavior, indicating a constant viscosity at different shear rates. This result was due to infrequent interactions between CNFs in the diluted suspensions under flow. The lower concentration suspensions were predicted to have two plateaus at low and high shear rates. As shown in Figure 3b, we observed a plateau at high shear rates but not at low shear rates, due to the torque limitation $(0.2 \mu \mathrm{N} \mathrm{m})$ of the rheometer.

The intrinsic viscosities, $[\eta]\left(\mathrm{mlg}^{-1}\right)$, of the CNFs were estimated from the concentration dependency of the viscosities at the Newtonian plateau according to

$$
[\eta]=\lim _{c \rightarrow 0} \eta_{\mathrm{red}}=\lim _{c \rightarrow 0}\left(\frac{\eta-\eta_{0}}{\eta_{0}} \cdot \frac{1}{c}\right)
$$

where $c$ is the concentration of the suspension $\left(\mathrm{g} \mathrm{ml}^{-1}\right), \eta_{\text {red }}$ is the reduced viscosity $\left(\mathrm{ml} \mathrm{g}^{-1}\right), \eta$ is the viscosity of the suspension $(\mathrm{Pa} \cdot \mathrm{s})$, and $\eta_{0}$ is the viscosity of the solvent $(\mathrm{Pa} \cdot \mathrm{s})$.

Figure 4 displays the $\eta_{\text {red }}$ values for concentrations of $<0.001 \mathrm{~g} \mathrm{ml}^{-1}(0.1 \mathrm{wt} \%)$ at a shear rate of $39.8 \mathrm{~s}^{-1}$ for the SCNFs and CNFs. This shear rate was chosen according to the previous report by Araki et al. ${ }^{2}$ The result for CNCs with small aspect ratios are included for comparison, even though the $\eta_{\text {red }}$ values for the CNCs suspensions were determined using a capillary viscometer. As the viscosities of the CNCs at concentrations of less than $0.001 \mathrm{~g} \mathrm{ml}^{-1}$ were very low, they could not be measured using the cone-plate

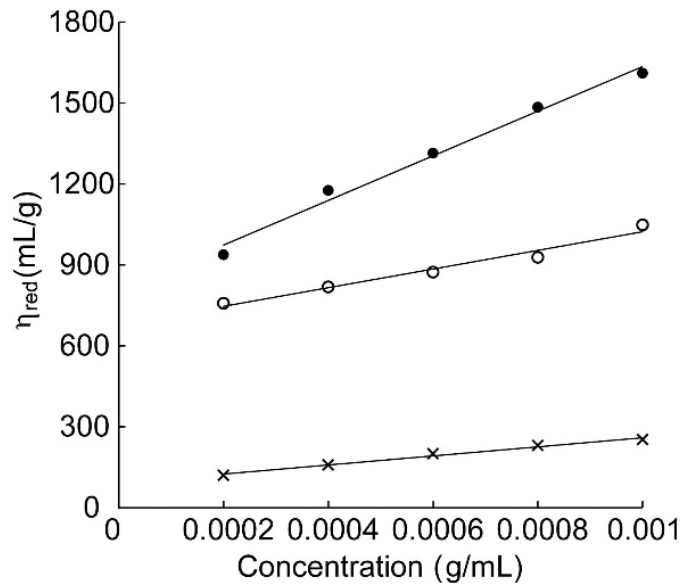

Figure 4 Reduced viscosity ( $\left.\eta_{\mathrm{red}}\right)$ of the cellulose nano-crystal (CNC) suspensions (cross, $\times$ ), shortened cellulose nanofiber (SCNF; open circle, $\mathrm{O})$, and cellulose nanofiber (CNF; filled circle, $\mathbf{~ ) ~ a t ~ l o w ~ c o n c e n t r a t i o n s . ~}$

rheometer. However, the relationship between viscosity and aspect ratio has already been reported for rod-shaped $\mathrm{CNCs}^{2,12}$ We attempted to apply this relationship for the SCNFs and CNFs with larger aspect ratio in our study.

The regression lines fit well for all of the suspensions, indicating a negligible effect of fiber-fiber interactions on the viscosities of the suspensions under these conditions. According to equation $1,[\eta]$ is obtained from the intercept of the regression line on the $y$ axis. The $[\eta]$ values for the CNFs, SCNFs and CNCs are listed in Table 1. Nanofibers with higher aspect ratios had larger $[\eta]$ values.

\section{Relationships between aspect ratios and intrinsic viscosity $[\eta]$ for} CNFs

The aspect ratios $(f)$ of the rigid rod-like particles were predicted theoretically from $[\eta]$ using the Simha equation ${ }^{14}$

$$
\frac{\eta-\eta_{0}}{\eta_{0} \phi}=[\eta] \cdot \rho=\frac{14}{15}+\frac{f^{2}}{5}\left\{\frac{1}{3(\ln 2 f-1.5)}+\frac{1}{\ln 2 f-0.5}\right\}
$$

where $\rho$ and $\phi$ represent the density $\left(\mathrm{g} \mathrm{cm}^{-3}\right)$ and volume fraction of the particles, respectively. Araki et al. ${ }^{2}$ and Boluk et al. ${ }^{12}$ demonstrated that the theoretical $f$ values and the actual value measured by morphological observation are similar for CNCs. The theoretical $f$ values for the CNCs, SCNFs and CNFs were calculated from the $[\eta]$ values (Table 1 ). The theoretical $f$ value for the CNCs corresponded well with the actual value measured by AFM. However, the theoretical $f$ values of the SCNFs and CNFs were lower than their actual $f$ values. This difference gradually became larger as $f$ increased.

Wierenga and Philipse ${ }^{15}$ summarized the $[\eta]$ values for particles and polymers with vast $f$ variations $(20-1000)$. They showed that $[\eta]$ was described by a function of $f$ using the Onsager equation, ${ }^{16}$ which provided almost the same results as the Simha equation ${ }^{14}$ (Figure 5) over a vast $f$ range. Furthermore, they stated that this prediction was accurate regardless of specific material properties such as chain flexibility, surface charge or attraction. Although most of the data in their paper $^{15}$ corresponded with the Onsager prediction, a $0.1 \mathrm{M}$ aqueous $\mathrm{NaCl}$ suspension of xanthan ${ }^{17}$ deviated from the prediction at $f$ values of $>100$ (Figure 5). Xanthan, an ionic polysaccharide, exists as a rigid double-stranded helix in aqueous salt solutions. ${ }^{18,19}$ The relationship between $[\eta]$ and the molecular weight of xanthan is well understood. To describe $[\eta]$ from the molecular weight, the rigidity of the molecular chains must be considered. Xanthan fits a wormlike 


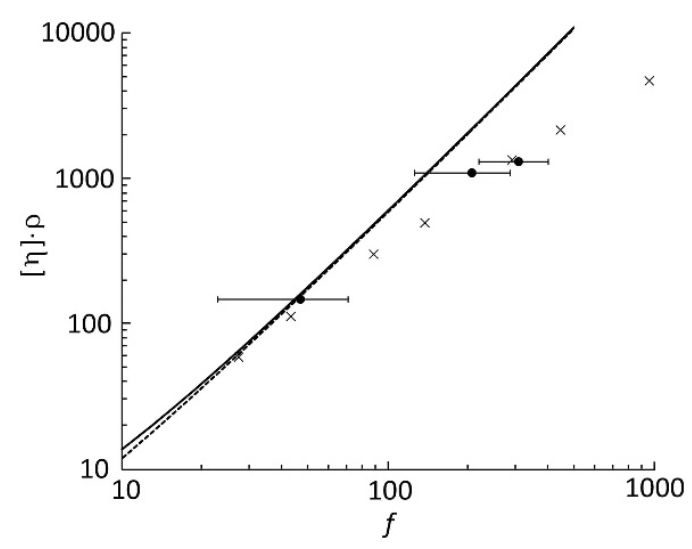

Figure 5 Relationship between intrinsic viscosity and aspect ratio for cellulose nanofibers (filled circle, $\bullet$ ) and xanthan ${ }^{17}$ (cross, $\times$ ) according to the Simha ${ }^{14}$ (solid line) and Onsager ${ }^{16}$ (broken line) predictions.

chain model, corresponding to its semi-flexible characteristics. ${ }^{20,21}$ Thus, when considering the relationship between $[\eta]$ and $f$ for xanthan, semi-flexible rods must be used rather than rigid rods.

The relationships between $[\eta]$ and $f$ for CNCs, SCNFs and CNFs are shown in Figure 5 . We observed a dependency of $[\eta]$ on $f$ for the CNCs, SCNFs and CNFs that was similar to xanthan. This finding indicated that the CNCs, SCNFs and CNFs exist as semi-flexible rods in water. In addition, their rigidity was supposed to be close to that of xanthan.

CNFs consisting of highly aligned molecules show remarkable crystallinity. However, Nishiyama et al. ${ }^{22}$ observed periodic disordered regions with a 150-nm cycle along the CNF axis using neutron scattering. This finding implies that the persistence length of CNFs is $150 \mathrm{~nm}$, if they are corresponded to the semi-flexible rods. As the CNCs existed as crystal bundles, their flexibility was very low even at lengths longer than the persistence length. For longer length CNFs and SCNFs with higher aspect ratios than the CNCs, significantly greater flexibility was observed, as demonstrated by their lower intrinsic viscosities compared with the theoretical values for rigid rods.

\section{CONCLUSIONS}

CNCs, SCNFs and CNFs were obtained from wood cellulose. The measured aspect ratios were highest for CNFs and lowest for CNCs. Intrinsic viscosity, $[\eta]$, was obtained by measuring the viscosity of CNC, SCNF and CNF suspensions at concentrations $<0.1 \mathrm{wt} \%$. The higher aspect ratio nanofibers showed larger $[\eta]$ values. The relationship between aspect ratio and $[\eta]$ for the CNCs agreed with the theoretical prediction for rigid rods; however, the relationships observed for the SCNFs and CNFs was not in agreement with the predictions. On the basis of a comparison with the $[\eta] /$ aspect ratio of xanthan, the CNFs used in our study can be considered to be semi-flexible rods rather than rigid rods.

\section{ACKNOWLEDGEMENTS}

This work was supported by Okayama Green-Bio Project run by Okayama Prefecture.

1 Marchessault, R. H., Morehead, F. F. \& Koch, M. J. J. Some hydrodynamic properties of neutral suspensions of cellulose crystallites as related to size and shape. Colloid Sci. 16, 327-344 (1961).

2 Araki, J., Wada, M., Kuga, S. \& Okano, T. Flow properties of microcrystalline cellulose suspension prepared by acid treatment of native cellulose. Colloids Surf. A: Physicochem. Eng. Aspects 142, 75-82 (1998).

3 Elazzouzi-Hafraoui, S., Nishiyama, Y., Putaux, J.-L., Heux, L., Dubreuil, F. \& Rochas, C. The shape and size distribution of crystalline nanoparticles prepared by acid hydrolysis of native cellulose. Biomacromolecules 9, 57-65 (2008).

4 Beck-Candanedo, S., Roman, M. \& Gray, D. G. Effect of reaction conditions on the properties and behavior of wood cellulose nanocrystal suspensions. Biomacromolecules 6, 1048-1054 (2005).

5 Saito, T., Nishiyama, Y., Putaux, J.-L., Vignon, M. \& Isogai, A. Homogeneous suspensions of individualized microfibrils from TEMPO-catalyzed oxidation of native cellulose. Biomacromolecules 7, 1687-1691 (2006).

6 Abe, K., Iwamoto, S. \& Yano, H. Obtaining cellulose nanofibers with a uniform width of $15 \mathrm{~nm}$ from wood. Biomacromolecules 8, 3276-3278 (2007).

7 Iwamoto, S., Abe, K. \& Yano, H. The effect of hemicelluloses on wood pulp nanofibrillation and nanofiber network characteristics. Biomacromolecules $\mathbf{9}$, 1022-1026 (2008)

8 Pääkkö, M., Ankerfors, M., Kosonen, $\mathrm{H}$., Nykänen, A., Ahola, S., Osterberg, M., Ruokolainen, J., Laine, J., Larsson, P. T., Ikkala, O. \& Lindström, T. Enzymatic hydrolysis combined with mechanical shearing and high-pressure homogenization for nanoscale cellulose fibrils and strong gels. Biomacromolecules 8, 1934-1941 (2007).

9 Henriksson, M., Henriksson, G., Berglund, L. A. \& Lindström, T. An environmentally friendly method for enzyme-assisted preparation of microfibrillated cellulose (MFC) nanofibers. Eur. Polym. J. 43, 3434-3441 (2007).

10 Shinoda, R., Saito, T., Okita, Y. \& Isogai, A. Relationship between length and degree of polymerization of TEMPO-oxidized cellulose nanofibrils. Biomacromolecules 13, 842-849 (2012).

$11 \mathrm{Li}$, Q. \& Renneckar, S. Molecularly thin nanoparticles from cellulose: isolation of sub-microfibrillar structures. Cellulose 16, 1025-1032 (2009).

12 Boluk, Y., Lahiji, R., Zhao, L. \& McDermott, M. T. Suspension viscosities and shape parameter of cellulose nanocrystals (CNC). Colloids Surf. A Physicochem. Eng. Aspects 377, 297-303 (2011).

13 Uetani, K. \& Yano, H. Semiquantitative structural analysis of highly anisotropic cellulose nanocolloids. ACS Macro Lett. 1, 651-655 (2012).

14 Simha, R. J. The influence of brownian movement on the viscosity of solutions. Phys. Chem. 44, 25-34 (1940).

15 Wierenga, A. M. \& Philipse, A. P. Low-shear viscosity of isotropic dispersions of (Brownian) rods and fibres; a review of theory and experiments. Colloids. Surf. A Physicochem. Eng. Aspects 137, 355-372 (1998).

16 Onsager, L. Viscosity and particle shape in colloid solutions. Phys. Rev. 40, 1028 (1932)

17 Takada, Y., Sato, T. \& Teramoto, A. Dynamics of stiff chain polymers in isotropic solution. 2. Viscosity of aqueous solutions of xanthan, a rigid double-helical polysaccharide. Macromolecules 24, 6215-6219 (1991).

18 Sato, T., Kojima, S., Norisuye, T. \& Fujita, H. Double-stranded helix of xanthan in dilute solution -further evidence. Polym. J. 16, 423-429 (1984).

19 Sato, T., Norisuye, T. \& Fujita, H. Double-stranded helix of xanthan: dimensional and hydrodynamic properties in $0.1 \mathrm{M}$ aqueous sodium chloride. Macromolecules 17, 2696-2700 (1984).

20 Yamakawa, H. \& Fujii, M. Intrinsic viscosity of wormlike chains. Determination of shift factor. Macromolecules 7, 128-135 (1974).

21 Yamakawa, H. \& Yoshizaki, T. Transport coefficients of helical wormlike chains. 3. Intrinsic viscosity. Macromolecules 13, 633-643 (1980).

22 Nishiyama, Y., Kim, U. J., Kim, D. Y., Katsumata, K., May, R. P. \& Langan, P. Periodic disorder along ramie cellulose microfibrils. Biomacromolecules 4, 1013-1017 (2003). 